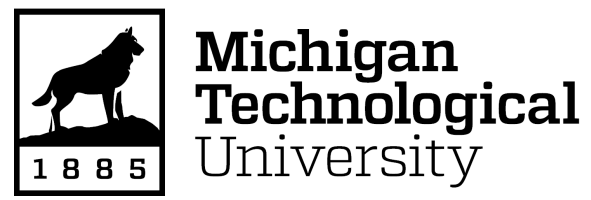

Michigan Technological University Digital Commons @ Michigan Tech

4-2003

\title{
Monte Carlo simulations of surface phase transitions in a modulated layered structure
}

\author{
Da Gao \\ Michigan Technological University \\ John A. Jaszczak \\ Michigan Technological University
}

Follow this and additional works at: https://digitalcommons.mtu.edu/physics-fp

Part of the Atomic, Molecular and Optical Physics Commons, and the Optics Commons

\section{Recommended Citation}

Gao, D., \& Jaszczak, J. A. (2003). Monte Carlo simulations of surface phase transitions in a modulated layered structure. Physical Review B, 67. http://dx.doi.org/10.1103/PhysRevB.67.155420

Retrieved from: https://digitalcommons.mtu.edu/physics-fp/26

Follow this and additional works at: https://digitalcommons.mtu.edu/physics-fp

Part of the Atomic, Molecular and Optical Physics Commons, and the Optics Commons 


\title{
Monte Carlo simulations of surface phase transitions in a modulated layered structure
}

\author{
Da Gao and John A. Jaszczak \\ Department of Physics, Michigan Technological University, Houghton, Michigan 49931 \\ (Received 22 July 2002; revised manuscript received 14 November 2002; published 30 April 2003)
}

\begin{abstract}
A solid-on-solid model of a layered crystal, which has five layers per repeat period in the direction normal to the surface and with only nearest-neighbor interactions, is studied using Monte Carlo simulation to investigate the relationship between crystal structure and the corresponding surface phases. Equilibrium properties, such as the surface specific heat, interface width, and autocorrelation times, are studied as a function of temperature and system size. Results indicate three distinct surface phases exist in this model: a lowtemperature flat phase, an intermediate-temperature disordered but flat phase, and a high-temperature rough phase. We suggest the possibility of introducing several intermediate phases, as well as a rough phase, in a single system by appropriate modulation of the periodicity of the crystal structure normal to the surface. At the same time, growth simulations show an interesting growth-induced smoothing in the intermediate phase where, at low supersaturations, the growing intermediate phase has a smaller interface width than it does in equilibrium.
\end{abstract}

DOI: 10.1103/PhysRevB.67.155420 PACS number(s): 68.35.Rh, 64.60.Cn, 64.60.Fr, 68.35.Bs

\section{INTRODUCTION}

As temperature is increased, a flat crystal surface may undergo different phase transitions leading up to roughening. Intermediate phases that can occur include reconstructed phases and a disordered flat (DOF) phase. ${ }^{1,2}$ The known mechanisms to realize the DOF phase include further-thannearest-neighbor interactions in solid-on-solid (SOS) models, ${ }^{1,3-7}$ and step-step interactions. ${ }^{8}$ Grimbergen et al. ${ }^{9}$ realized a DOF phase in a model with connected nets having different bond energies which lead to different step energies. Some examples of layered structures that are capable of stabilizing the DOF phase are: $\mathrm{CsCl}\{001\},{ }^{10} \mathrm{Si}\{111\},{ }^{11}$ and $\mathrm{Ge}$ $\{001\} .{ }^{12}$

In most models studied to date, the Hamiltonian is fairly complex. On the other hand, the existence of preroughening of diamond-cubic $\{111\}$ surfaces with only nearest-neighbor interactions remains somewhat controversial. ${ }^{11,13}$ In this study, we employ a relatively simple layered structure in a SOS model to investigate the relationship between crystal structure and surface phases and transitions. Without considering further-than-nearest-neighbor interactions, we find three clearly distinct phases: flat, intermediate, and rough, using Monte Carlo simulation. The transition from flat to intermediate in our model appears to be Ising-like. The intermediate phase is similar to a DOF phase in the sense that it has short-range transverse disorder but long-range flatness. In contrast to many other simulation studies with a DOF phase, the transition from flat to intermediate in this study is well separated in temperature from the roughening transition. We compare simulation results for various equilibrium properties and autocorrelation times with those of the well-known simple crystals model (SCM) or Kossel crystal. We also find an interesting growth-induced smoothing in the intermediate phase where, at low supersaturations, the growing intermediate phase has a smaller interface width than it does in equilibrium.

\section{DESCRIPTION OF THE MODEL}

Our model is a modified solid-on-solid (SOS) model with only nearest-neighbor interactions and unrestricted height differences between columns. As usual, no overhangs and vacancies are allowed. To realize a nontrivial layer stacking normal to the interface we follow Ref. 14 in which a layered quasiperiodic structure and a related entropic crystal model (ECM2) were investigated. In these models, there are two kinds of layers: One is called a "bond-cost" layer because it will cost energy when the interface wanders through such a layer. The other is called a "free layer." The interface can cross a free layer at no additional energy cost. The interface is described by integer heights $h(x, y)$, which is measured by the total number of layers in a column at $(x, y)$ relative to the height of a reference layer, and includes both bond-cost layers and free layers. Results from Ref. 14 show a series of surface transitions as a function of temperature in the quasiperiodic structure that increasingly disorders the surface, although the surface does not actually roughen (i.e., the surface width does not diverge with system size at any finite temperature). These results suggest that a similar but nontrivial periodicity of a layered structure could possibly lead to preroughening in a crystal model. In order to capture the local structure of the quasiperiodic structure in a periodic system, a different layered crystal model (LCM) (Fig. 1) was chosen to have a periodicity of five layers. In each period there are three bond-cost layers and two free layers.

The simulations are carried out using standard Metropolis Monte Carlo. A Monte Carlo move consists of attempting to change the height $h$ of a column by \pm 1 . One Monte Carlo sweep (MCS) transpires as a convenient unit of time when the number of attempted Monte Carlo moves is equal to the total number of columns. The change in surface energy is calculated by

$$
E=\frac{J}{2} \sum_{\langle i, j\rangle}\left|L\left(h_{i}\right)-L\left(h_{j}\right)\right|
$$




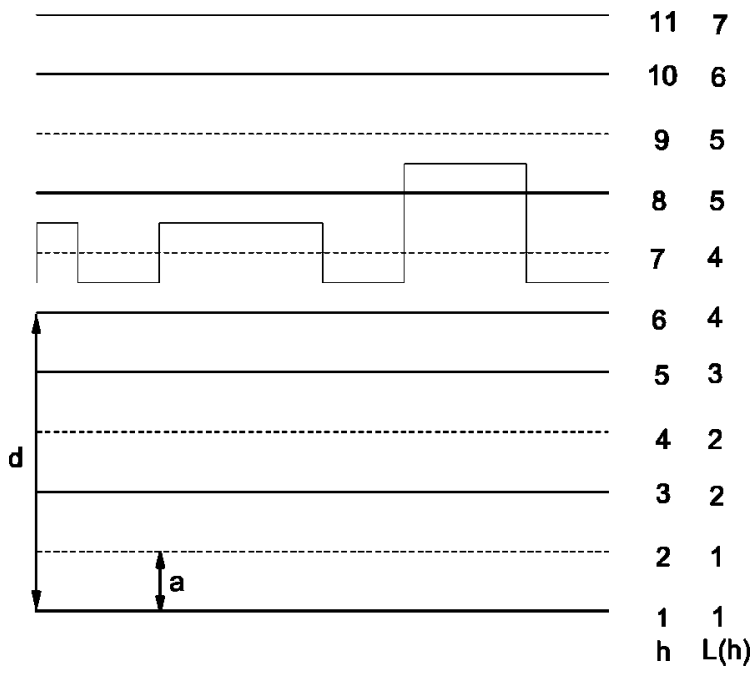

FIG. 1. Schematic of the structure of the LCM. The bold solid lines represent the bond-cost layers and the dashed lines represent the free layers. The unit cell height $d$ of this structure is equal to $5 a$ where $a$ is the unit layer distance. The fluctuating solid line shifted up by $0.5 a$ for clarity is a sample low-temperature interface whose boundaries are pinned to produce a step.

where $\langle i, j\rangle$ denotes that the sum is over all distinct nearestneighbor column pairs. For a given height $h, L(h)$ is the corresponding height of a column in terms of bond-cost layers only, $\left|L\left(h_{i}\right)-L\left(h_{j}\right)\right|$ counts broken horizontal bonds, and the energy per bond is $J$. The periodic structure in Fig. 1 is given by the relationship

$$
h(L)= \begin{cases}{\left[\frac{5 L(h)}{3}\right]} & \text { for } L(h) \geqslant 0, \\ {\left[\frac{5 L(h)-2}{3}\right]} & \text { for } L(h)<0,\end{cases}
$$

where $[\cdots]$ denotes the greatest integer function.

\section{MONTE CARLO SIMULATION RESULTS}

Simulations were performed for several system sizes up to $N \equiv L \times L=131 \times 131$ column sites, with $L$ defined as the number of columns on one side of the surface, and for temperatures ranging from $k_{B} T / J=0.3$ up to 1.6 where $k_{B}$ is the Boltzmann constant. For each simulation, $10^{6}$ MCS were carried out for equilibration and data were taken for an additional $2 \times 10^{6}$ MCS except for the largest system size 131 $\times 131$ which had at least $10^{6}$ MCS for equilibration followed by $4 \times 10^{6}$ MCS for taking data. Autocorrelation times, defined below, were monitored in order to properly estimate error bars.

\section{A. Specific heat}

The surface specific heat $C$ is thermodynamically related to the second derivative of the surface free energy, and is conveniently calculated from energy fluctuations:

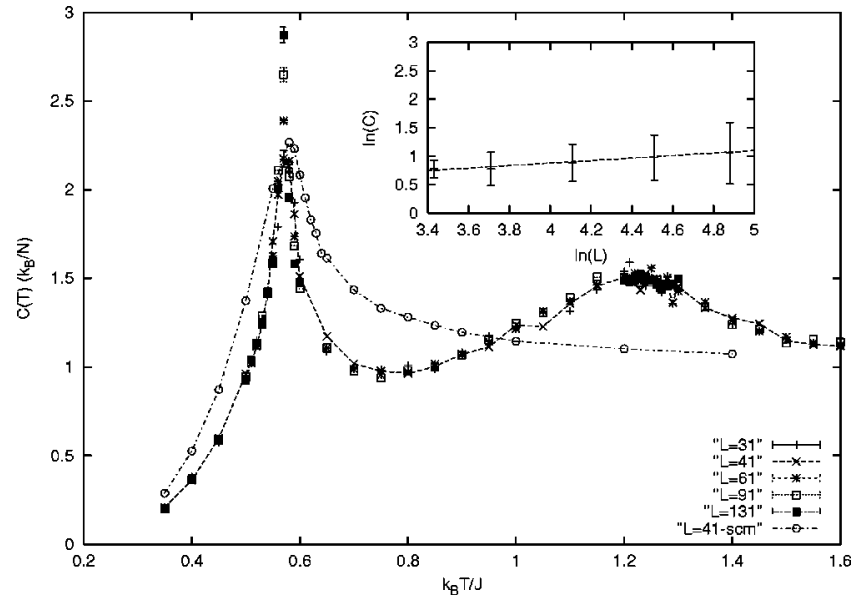

FIG. 2. Surface specific heat (in units of $k_{B}$ per column where $k_{B}$ is the Boltzmann constant) versus temperature for the LCM with different size surfaces $(L=31,41,61,91,131)$. Also compared with SCM (simple crystal model) case with $L=41$. The inserted graph is the natural logarithm of specific heat versus the natural logarithm of surface size for the LCM near the first peak $\left(k_{B} T_{C} / J=0.57\right)$.

$$
C(T)=\frac{\left\langle E^{2}\right\rangle-\langle E\rangle^{2}}{N k_{B} T^{2}},
$$

where $E$ is the surface energy and $\langle\cdots\rangle$ represents an ensemble average. Due to its sensitivity to energy fluctuations in finite systems, specific heat can be a useful tool for indicating possible phase transitions.

The surface specific heat as functions of temperature for the LCM as well as a simple crystal model (SCM) are shown in Fig. 2. In a simple crystal model all the bonds are same and therefore there is only one kind of layer. There are two peaks in the specific heat for the LCM while only one peak is observed for the SCM model. From Fig. 2 we observe that for the LCM the first peak near $k_{B} T / J=0.6$ shows a clear finite-size dependence while the second one near $k_{B} T / J$ $=1.2$ has no such dependence. This suggests that the second peak is not a strong phase transition whereas the first peak may be. Finite-size scaling of the LCM specific heat (Fig. 2 inset), at the first peak $\left(k_{B} T_{C} / J=0.57\right)$, assuming that it diverges with system size as

$$
C \sim L^{\alpha / \nu},
$$

yields $\alpha / \nu=0.183 \pm 0.271$. The data fit equally well a logarithmic size dependence for the specific heat, which is consistent with the first peak corresponding to a twodimensional (2D) Ising transition.

Presuming that the second peak near $k_{B} T / J=1.26$ is a Kosterlitz-Thouless (KT)-type roughening transition, we next investigate the interface width as a function of temperature and system size.

\section{B. Surface width and fluctuations}

The square of the surface width $W^{2}$ is a useful tool to identify the roughening temperature of the surface, and is defined as

$$
W^{2} \equiv \frac{1}{N} \sum_{i}\left\langle\left(h_{i}-\bar{h}\right)^{2}\right\rangle
$$




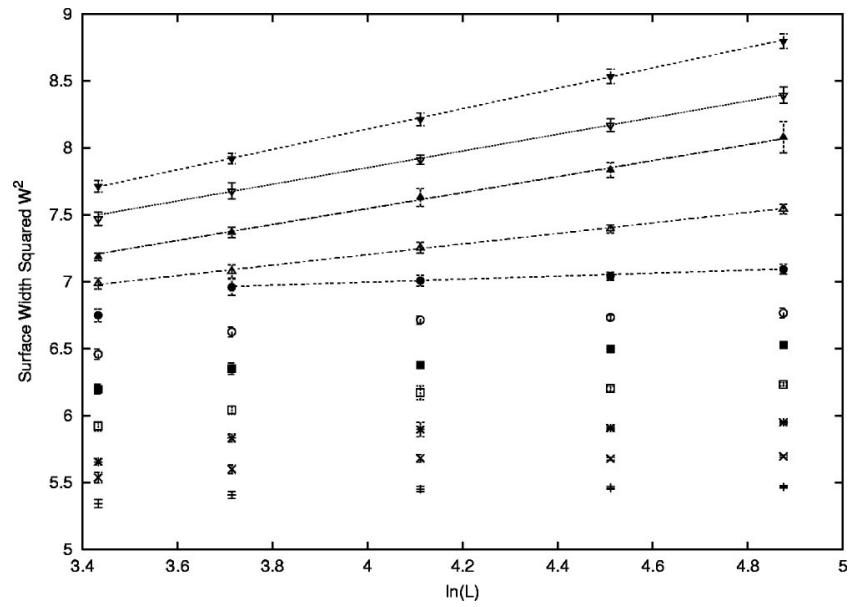

FIG. 3. Surface width squared versus natural logarithm of surface size for different temperatures near $T_{R}$ (roughening temperature) for LCM. From bottom to top $k_{B} T / J$ $=1.20,1.21,1.22,1.23,1.24,1.25,1.26,1.27,1.28,1.29,1.30$. Straight lines represent least-squares fits to the data.

where $\bar{h}$ is the average surface height in a particular configuration and the sum is over all columns. For large $L$ the interface width saturates to a constant value for temperatures below $T_{R}$, and logarithmically diverges with the system size $L$ for $T>T_{R}$, the roughening temperature:

$$
W^{2} / a^{2} \approx K(T) \ln (L) \quad \text { for } \quad T \geqslant T_{R}
$$

We can locate $T_{R}$ via the characteristic Kosterlitz-Thouless behavior of $K(T),{ }^{15}$

$$
K(T)= \begin{cases}\frac{1}{\pi^{2}} \quad \text { for } T=T_{R}, \\ \frac{1}{\pi^{2}}+C\left(T-T_{R}\right)^{1 / 2} \quad \text { for } T \rightarrow T_{R}^{+},\end{cases}
$$

where $C$ is a nonuniversal constant, while the value $K\left(T_{R}\right)$ and the power $\frac{1}{2}$ are universal features. Figure 3 shows the results of the interface width squared $W^{2}$ versus logarithm of system size for temperatures near the second peak of Fig. 2. From the graph we note that for $k_{B} \mathrm{~T} / \mathrm{J} \leqslant 1.25, W^{2}$ shows no sign of diverging. On the other hand, for $k_{B} T / J>1.25, W^{2}$ is linear in $\ln (L)$ for $L \geqslant 41$. Following Ref. 4, we identify $k_{B} T_{R} / J=1.260 \pm 0.001$ by interpolating the temperature at which $K(T)$ takes on the universal value $1 / \pi^{2}$.

In order to compare qualitatively what happens to the surface in the flat, intermediate and rough phases more clearly, Fig. 4 presents graphs of one-dimensional vertical cuts through two-dimensional surfaces of the LCM for system size $L=61$ at three different representative temperatures. From Fig. 4 it can be seen that in the flat phase the interface fluctuations are bounded between bond-cost layers but freely cross the free layer. In the intermediate phase, just above $T_{C}$, the surface fluctuates through three layers (one energy-cost layer and two free layers) but is bounded by two pairs of adjacent energy-cost layers [Fig. 4(b)]. Finally, above the roughening transition the interface can cross any layer as illustrated in Fig. 4(c). Using Fig. 4 we can estimate the average height of the surface in the intermediate phase by assuming that the columns have equal probability to be at $h=-1,-2,-3$, and -4 between pairs of energy-cost layers. The estimated value is -2.5 , which is in good agreement with the results of Fig. 5 for $T_{C}<T<T_{R}$. We also show the temperature dependence of the average surface height for LCM with $L=61$ in Fig. 5. There is an abrupt shift by one layer ( $1 / 5$ of the period) in the average height of the surface at $T_{C}$ where the surface changes from being flat (confined between bond-cost layers but with a free layer in between) to intermediate (confined between two pairs of adjacent bond-cost layers), consistent with Figs. 4(a) and (b). At $T_{C}$ itself there is large negative peak, which is simply a large fluctuation consistent with 2D Ising behavior.

\section{Critical slowing down}

Swendsen ${ }^{16,17}$ has reported the existence of critical slowing down at the roughening transition in Monte Carlo simu-
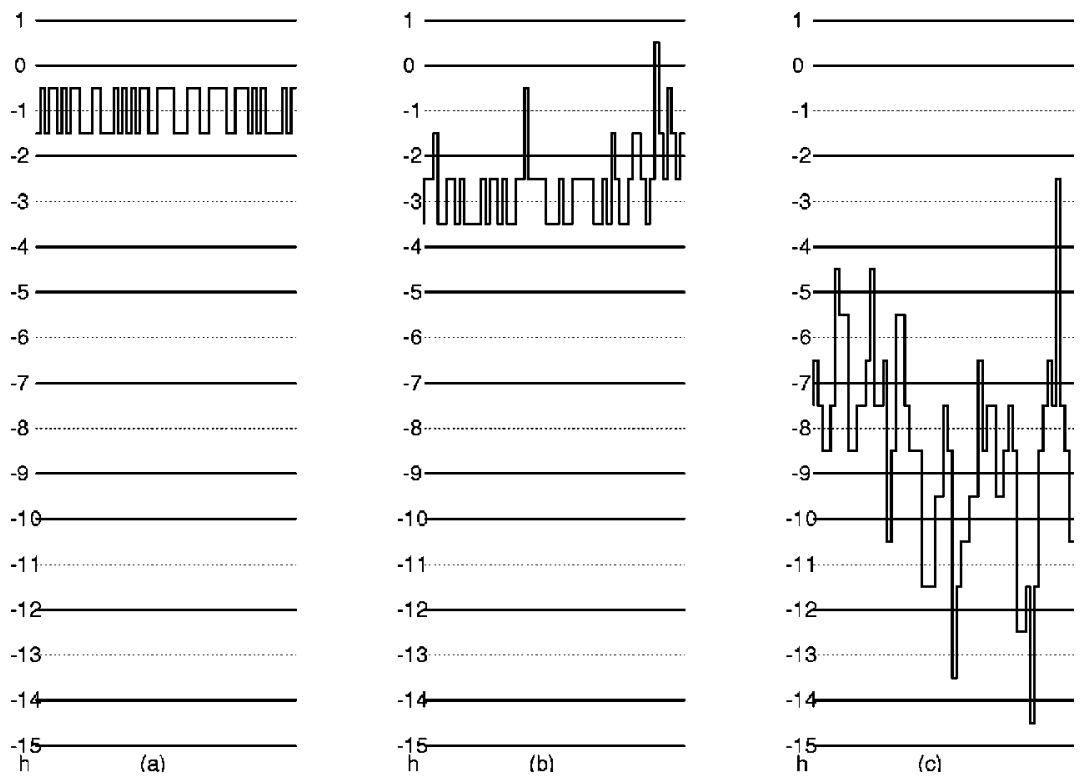

FIG. 4. One-dimensional vertical cuts through typical twodimensional surfaces superimposed on the LCM structure at (a) $k_{B} T / J=0.400$, (b) $k_{B} T / J=0.580$, and (c) $k_{B} T / J=1.290$. The system size is $L \times L=61 \times 61$. The vertical axes numbers are column heights measured from an energycost layer arbitrarily numbered zero. For clarity, the surface structure is drawn shifted by $+0.5 a$ where $a$ is the unit layer distance. 


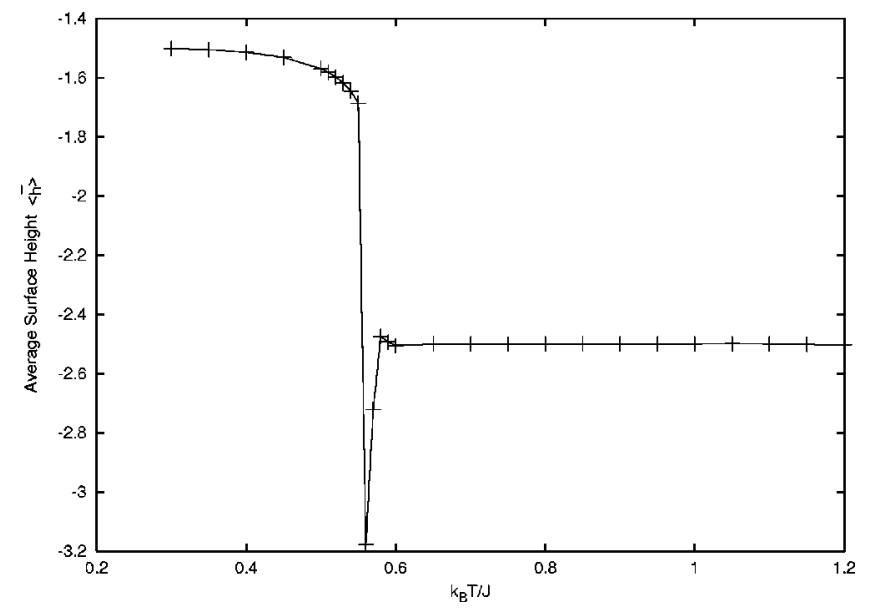

FIG. 5. Average surface height versus temperature for our LCM with a system size $L=61$ near $k_{B} T_{C} / J=0.570$.

lations of a solid-on-solid model of a Kossel crystal. In our Monte Carlo simulations of the LCM we find that the critical slowing down exists near both the roughening transition and the lower-temperature Ising-like transition. To study critical slowing down we investigate autocorrelation times for various quantities as a function of temperature and system size. For a fluctuating quantity, $F(t)$, the normalized autocorrelation function is

$$
A_{F}(t) \equiv \frac{\langle F(t) F(0)\rangle-\langle F\rangle^{2}}{\left\langle F^{2}\right\rangle-\langle F\rangle^{2}},
$$

Following Swendsen ${ }^{16,17}$ we calculate the autocorrelation time $\tau$ two ways. One is called the long autocorrelation time $\tau_{l F}$, which is determined approximately from the time when $A_{F}(t)$ drops to less than a few percent. On the other hand, the short autocorrelation time $\tau_{s F}$ is calculated from

$$
\tau_{s F}=-\frac{1}{\ln \left[A_{F}(t=1)\right]} .
$$

Autocorrelation times for both the surface energy and the surface width squared for the LCM are shown in Figs. 6-9. Corresponding autocorrelation times for the SCM are also shown for comparison. Since the surface energy and the surface width squared are recorded in MCS the unit of corresponding autocorrelation time is also MCS.

Both long and short autocorrelation times for $E$ and $W^{2}$ at $T_{c}$ were observed to scale ${ }^{18,19}$ with system size as

$$
\tau \sim L^{z}
$$

as shown in Fig. 10. Least-squares fits of these data yield the dynamic critical exponents $z$ given in Table I.

From Table I we observe a significant difference between the long and short dynamical critical exponent $z$, even for the same physical quantity, $E$ or $W^{2}$. However, the dynamical critical exponent of $E$ is always smaller than that of $W^{2}$ for both long and short autocorrelation times as may be expected since surface width is more sensitive to long-range correlations although it is claimed to be the same for all observables

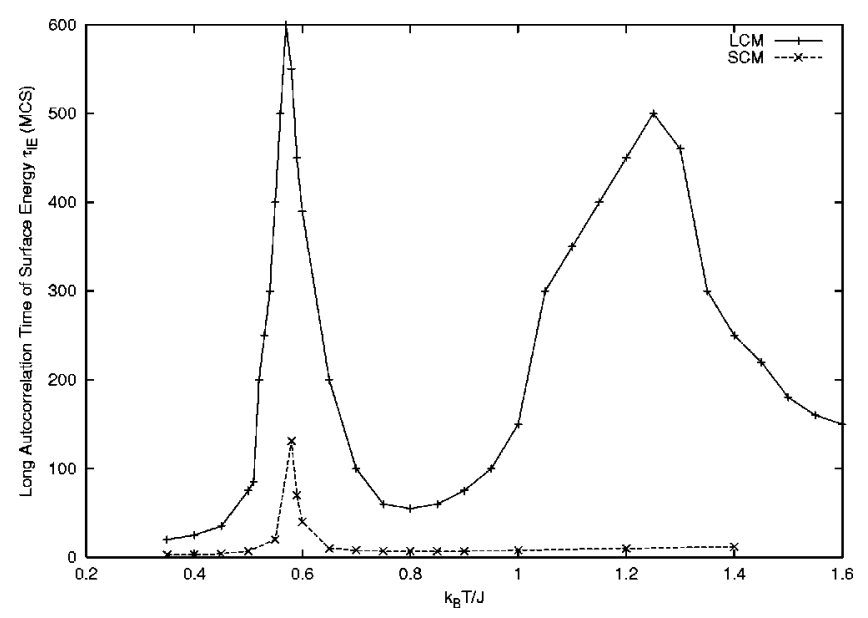

FIG. 6. Comparison of the long autocorrelation time of the surface energy between the LCM and SCM. The size of both systems is $L=61$.

in Ref. 19. At the same time we calculate $z$ for surface width squared at $T_{R}$ and the value is $0.70 \pm 0.08$. Due to large error bars we did not obtain reliable $z$ values at $T_{R}$, but they were smaller than the values at $T_{C}$.

\section{DISCUSSION AND CONCLUSIONS}

We first discuss the interface width here in more detail. Theoretically the interface width $W \equiv \sqrt{W^{2}}$ defined by Eq. (5) will show size dependence if $T \geqslant T_{R}$ and as size becomes infinite the interface width will diverge. ${ }^{4,10,20}$ Figure 11 shows the interface width $W$ versus temperature with several different system sizes for the LCM, as well as results for two sizes $(L=21,41)$ for the SCM. The interface width $W$ does depend on the system size once the temperature is above the respective $T_{R}$ values for both LCM and SCM as expected., At $T_{C}$ the interface width $W$ increases sharply and once in the intermediate phase it becomes smooth and size independent until $T_{R}$. From the inset of Fig. 11 we note that at $T_{C}$ itself, $W^{2}$ appears to diverge logarithmically with system

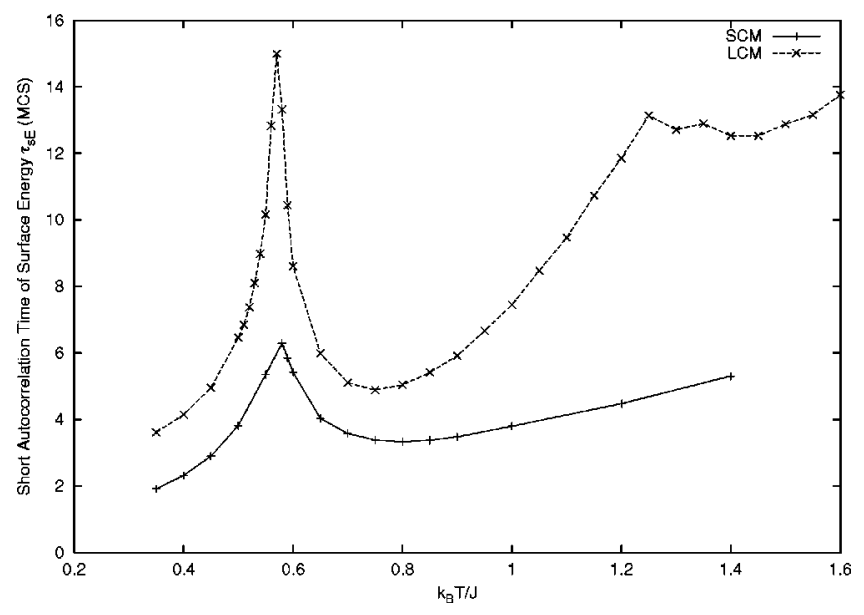

FIG. 7. Comparison of the short autocorrelation time of the surface energy between the LCM and SCM. The size of both systems is $L=61$. 


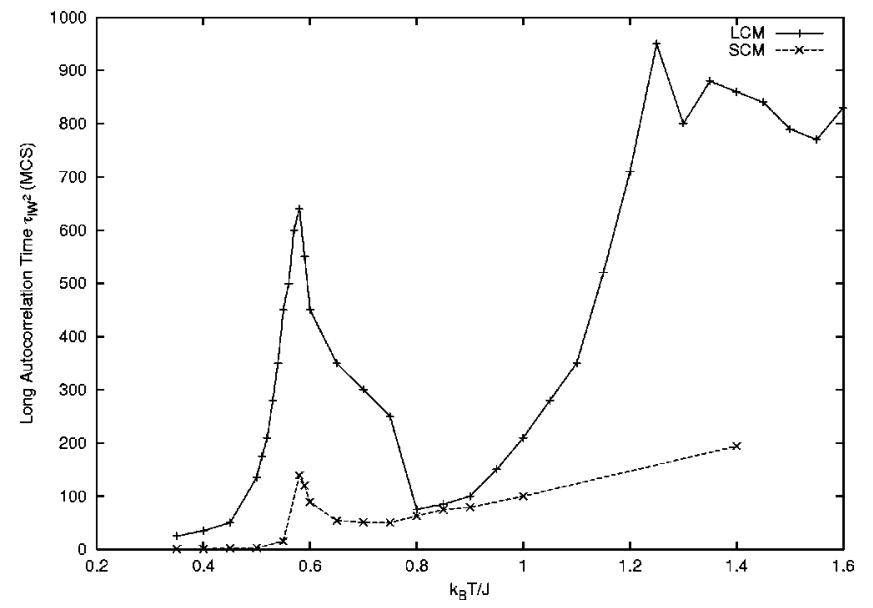

FIG. 8. Comparison of the long autocorrelation time of the surface width squared between the LCM and SCM. The size of both systems is $L=61$.

size, at least until $L \approx 400$. At larger $\ln (L)$, however, the divergence seems to be slowing down. Furthermore, even for $L=601, W^{2}$ is less than 1.25 , which indicates that the surface fluctuations are still confined between two pairs of adjacent bond-cost layers (despite relatively large " $L$ "). Although not conclusive, these results also suggest that the transition at $T_{C}$ is $2 \mathrm{D}$ Ising-like, in contrast with other preroughening transitions which are rough right at the preroughening temperature. ${ }^{3,20,21}$

Before the Ising-like transition, the surface can only fluctuate across one free layer; however, once in the intermediate phase it can wander through one energy-cost layer plus two free layers (three layers total), but still is bounded between two pairs of adjacent energy-cost layers [i.e., between the $h$ $=0,1$ pair and the $h=-4,-5$ pair in Fig. 4(b)]. Accordingly, the interface width of the intermediate phase should be nearly triple of that of the previous ordered flat (OF) phase. Based on Fig. 4, we can estimate the interface width of the intermediate phase as follows:

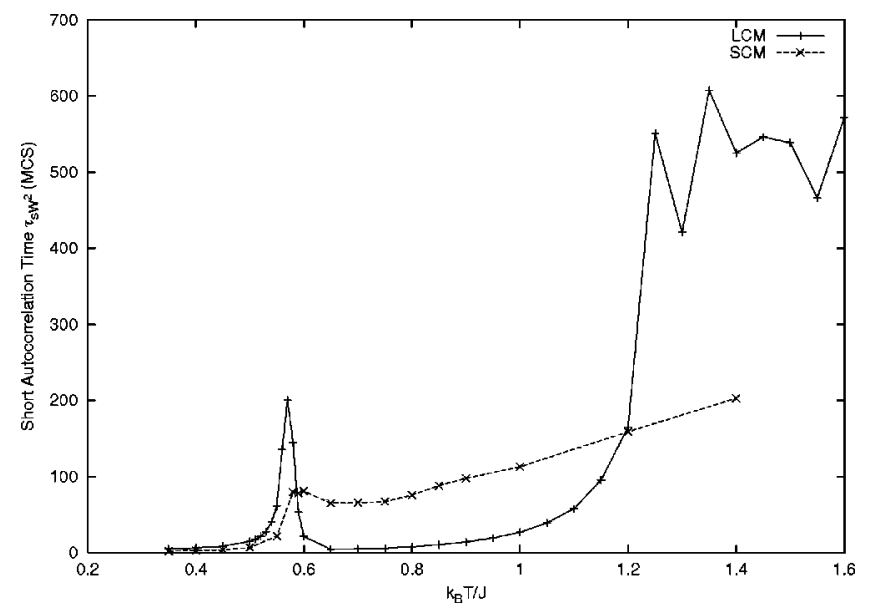

FIG. 9. Comparison of the short autocorrelation time of the surface width squared between the LCM and SCM. The size of both systems is $L=61$.

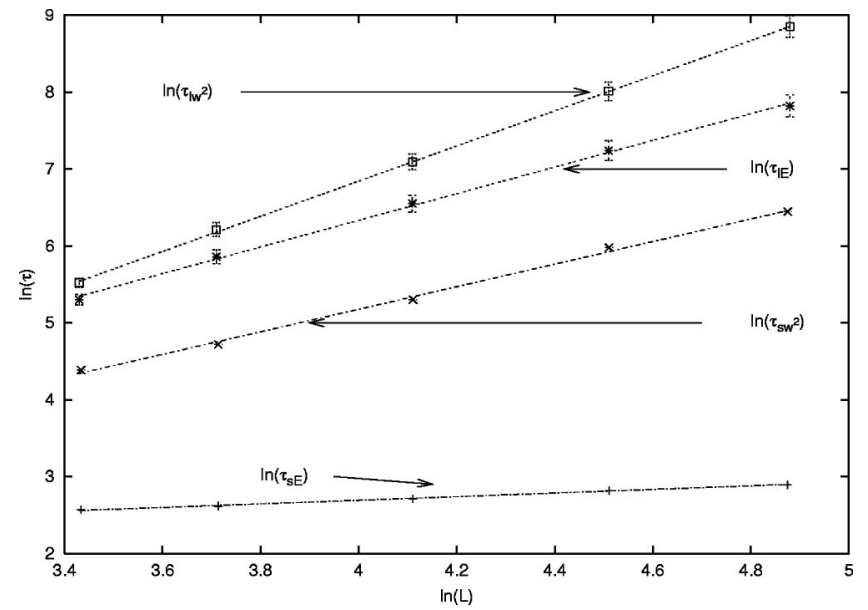

FIG. 10. Natural logarithm of the autocorrelation times for the surface energy and surface width squared including long and short cases (see text), as specified in the graph via arrows, versus natural logarithm of surface size for the LCM at $k_{B} T_{C} / J=0.57$.

$$
W_{\text {intermediate }}=\sqrt{\bar{h}^{2}-\bar{h}^{2}}=1.7,
$$

if we assume equal, nonzero probabilities for the surface to occupy any of the six layers between the bonding layers $h$ equals 0 and -5 , and zero probability elsewhere. Similarly,

$$
W_{O F}=\sqrt{\overline{h^{2}}-\bar{h}^{2}}=0.5,
$$

if we assume equal, nonzero probabilities for the surface to occupy only two layers between $h=-1$ and -2 , and zero probability elsewhere. Both values are in good agreement with the results shown in Fig. 11. Then we can calculate the ratio via these values,

$$
\frac{W_{\text {intermediate }}}{W_{O F}} \approx \frac{1.7}{0.5}=3.4,
$$

which is approximately the value we predicted.

Calculations of the autocorrelation times for the surface energy and the surface width squared indicate critical slowing down in two distinct temperature regions, corresponding to the two distinct phase transitions. Dynamical critical exponents $z$ were computed at $T_{C}$ and $T_{R}$. Values of $z$ at $T_{R}$ were found to be much smaller than at $T_{C}$ indicating that simulations at the Ising-like transition need extra care to generate accurate results. We are not aware of any reported $z$ values for interface width squared. On the other hand, compared to published values ${ }^{18,19}$ of $z \approx 2$ for the $2 \mathrm{D}$ Ising model, the $z$ values from the long autocorrelation times seem

TABLE I. Best-fit values for the long and short dynamical critical exponents for surface energy and surface width squared at $T_{C}$.

\begin{tabular}{cc}
\hline \hline Autocorrelation time & $z$ \\
\hline$\tau_{l E}$ & $1.73 \pm 0.04$ \\
$\tau_{l W^{2}}$ & $2.28 \pm 0.02$ \\
$\tau_{s E}$ & $0.235 \pm 0.008$ \\
$\tau_{s W^{2}}$ & $1.47 \pm 0.04$ \\
\hline \hline
\end{tabular}




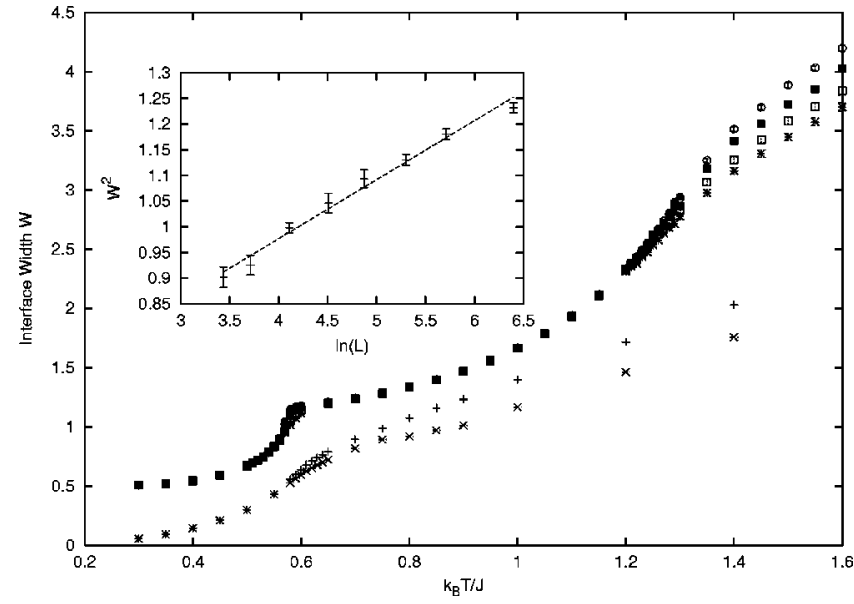

FIG. 11. Comparison of the interface width versus temperature among different system sizes $(L=31,41,61,91,131)$ for LCM. We also plot the SCM case with system size $L=21,41$. In the graph from top to bottom are $L=131,91,61,41,31 \mathrm{LCM}$ and then $L$ $=41,21 \mathrm{SCM}$. For the $L=131$ case we plot only the points near $T_{C}$ and $T_{R}$. The inset shows the linear dependence of $W^{2}$ on $\ln (L)$ at $k_{B} T_{C} / J=0.57$ for system sizes $L$ up to $L=601$.

to be consistent with $T_{C}$ corresponding to a $2 \mathrm{D}$ Ising transition, while the $z$ value from both short autocorrelation times, especially for the energy, seem rather small.

As a final check of the apparent 2D Ising nature of the transition at $T_{C}$, we performed dynamical simulations, following Refs. 3, 21, and 22, to check if growth is layer by layer or continuous at $T_{C}$. We investigated various chemical potential driving forces $\Delta \mu / J$ between 0.03 and 0.12 . At all temperatures investigated, $0.50 \leqslant k_{B} T / J \leqslant 0.75$, Monte Carlo simulation results showed no evidence of continuous growth behavior near $T_{C}$ at low supersaturations. Again, this result suggests that the transition at $T_{C}$ is $2 \mathrm{D}$ Ising-like, and is in contrast with preroughening results of Refs. 21 and 3. Interestingly, as shown in Fig. 12, the growth simulations show a growth-induced smoothing in the intermediate phase where, at low supersaturations, the growing intermediate phase has a smaller interface width than it does in equilibrium. Each peak in the interface width squared during growth occurs due to the nucleation and growth of a step in the system. Note that the growing interface and the equilibrium interface start at the same interface width, but the growing interface quickly "smooths" to a smaller interface width until a step nucleates on the surface. The terraces of the growing surface in the intermediate phase therefore are smoother than the surface would be in equilibrium. An analogous effect takes place in the Ising model where the application of an external applied magnetic field reduces magnetization fluctuations above the critical temperature. ${ }^{23}$ This effect should have interesting implications for understanding surface diffusion in such systems. The growth-induced-smoothing results from the combined effects of a vanishing step energy to cross the isolated bond-cost layer in the intermediate phase and the positive $\Delta \mu$, which causes the addition of particles in the intermediate phase (note the initial transient of increasing average surface height in Fig. 12). The pair of adjacent bondcost layers remains a nucleation barrier to growth since it has a nonzero step energy.

In a model with only nearest-neighbor interactions, we have found two distinct transitions separating three phases: flat, intermediate, and rough. Whereas the higher temperature transition is traditional Kosterlitz-Thouless-type roughening, the lower-temperature preroughening transition separating the flat and intermediate phases appears to be 2D Ising-like. In other model systems with a DOF phase, an Ising transition separates the DOF phase only from a reconstructed flat phase. On the other hand, Mazzeo et $a l .{ }^{24}$ have found flat and DOF phases separated by an Ising transition in a twocomponent crystal of a $\mathrm{CsCl}$ structure.

The existence of the distinct phases is due to the layering of the crystal structure. The sequence of energy cost layers and free layers modulates the surface energy and entropy in such a way as to allow the step free energy of steps with a height less than the period to vanish at a temperature below the roughening temperature. At the same time, the average height of the surface is able to jump by a fraction of the
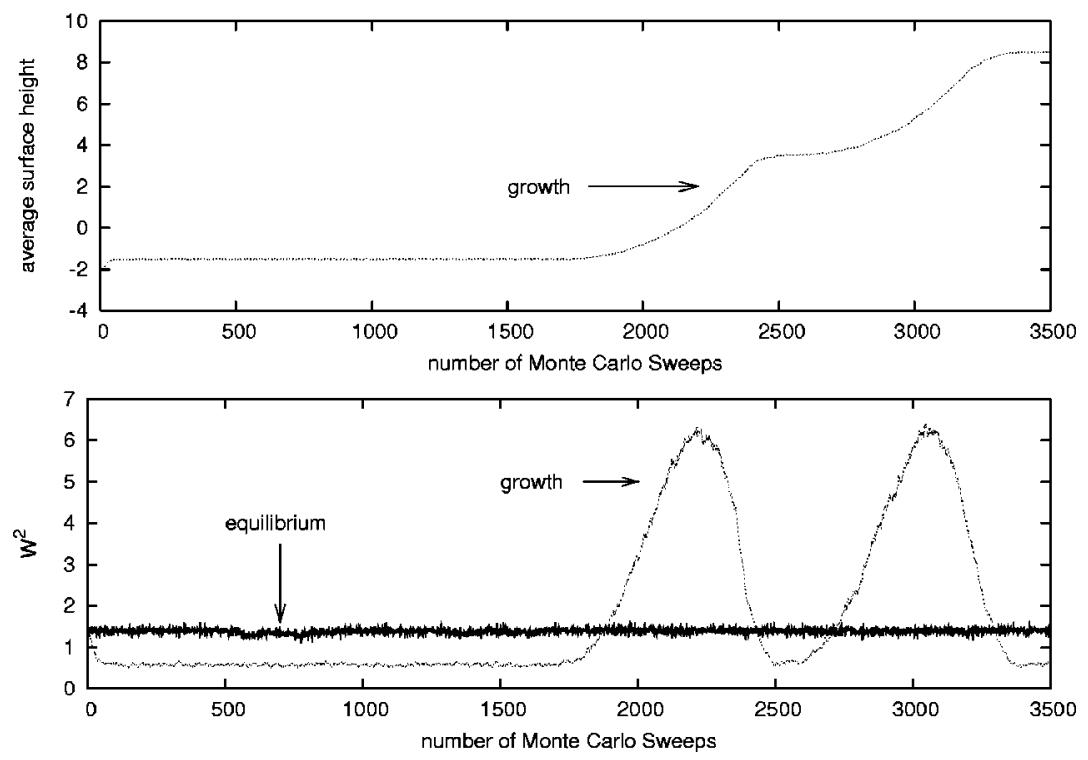

FIG. 12. Comparison of the interface width squared versus time (in units of MCS) between the growth and the equilibrium for the LCM in the intermediate phase, with system size $L=61$ and $k_{B} T / J=0.600$. The chemical potential driving force for growth is $\Delta \mu / J=0.08$. 
period. A similar behavior was suggested by the quasiperiodic structure investigated in Ref. 14 where several peaks were observed in the surface specific heat but which did not appear to roughen at finite temperature. On the other hand, the ECM2 mentioned earlier showed only one broad peak in the surface specific heat (Jaszczak and Kalogerakos, unpublished). While this model may have had both roughening and preroughening transitions, they were not distinct. In contrast to the LCM, the ECM2 does not have a unit cell large enough to allow for a shift in the average height by a fraction of the period as the step energy becomes smaller. Furthermore, the LCM has a twofold degeneracy for the ordered flat phase. Below the $T_{C}$ the ordered flat surface is localized around either of the two free layers within a period, and is thus asymmetrically localized within the unit cell. Above $T_{C}$ surface shifts its average height and becomes symmetrically localized between two pairs of adjacent energy cost layers in the intermediate phase. On the other hand, the ordered flat surface in the ECM2 has no degeneracy and can be localized only symmetrically within the unit cell of its structure. We expect therefore that it may be possible to engineer layered structures to exhibit two or more intermediate phases before the rough phase. It will also be interesting to investigate the effect of modulating the energies of the bond-cost layers. For example, if the energy of the bond-cost layer that is surrounded by free layers is $J$, the energies of each of the adjacent bond-cost layers could be reduced to $J^{\prime}$ such that $J / 2$ $<J^{\prime}<J$. While this would probably lead to a lowering of the roughening transition temperature, it would probably not increase the flat-to-intermediate transition temperature. Such a change should facilitate fluctuations of the intermediate phase beyond the double bond-cost layers, and perhaps change the 2D Ising transition to a preroughening transition. These results may also have implications for grain boundary roughness and grain growth since grain boundaries are interfaces in systems that can have complex but typically periodic interfacial energy modulations in the direction normal to the interface. ${ }^{25}$

\section{ACKNOWLEDGMENT}

We are grateful to Steve Hackney for insightful discussions. This work was supported in part through a grant from the National Science Foundation (EIA-9871133).
${ }^{1}$ K. Rommelse and M. den Nijs, Phys. Rev. Lett. 59, 2578 (1987).

${ }^{2}$ M. den Nijs and K. Rommelse, Phys. Rev. B 40, 4709 (1989).

${ }^{3}$ S. Prestipino, G. Santoro, and E. Tosatti, Phys. Rev. Lett. 75, 4468 (1995).

${ }^{4}$ G. Mazzeo, G. Jug, A.C. Levi, and E. Tosatti, Surf. Sci. 273, 237 (1992).

${ }^{5}$ G. Mazzeo, G. Jug, A.C. Levi, and E. Tosatti, Phys. Rev. B 49, 7625 (1994).

${ }^{6}$ P.J.M. Bastiaansen and H.J.F. Knops, Phys. Rev. B 53, 126 (1996).

${ }^{7}$ P.J.M. Bastiaansen and H.J.F. Knops, J. Chem. Phys. 104, 3822 (1996).

${ }^{8}$ R.F.P. Grimbergen, H. Meekes, and P. Bennema, Phys. Rev. B 58, 5258 (1998).

${ }^{9}$ R.F.P. Grimbergen, P. Bennema, and H. Meekes, Acta Crystallogr., Sect. A: Found. Crystallogr. A55, 84 (1999).

${ }^{10}$ G. Mazzeo, E. Carlon, and H. van Beijeren, Phys. Rev. Lett. 74, 1391 (1995).

${ }^{11}$ D.L. Woodraska and J.A. Jaszczak, Phys. Rev. Lett. 78, 258 (1997).

${ }^{12}$ M. den Nijs, J. Phys. A 30, 397 (1997).
${ }^{13}$ P. van Beurden, E. van Veenendaal, W.J.P. van Enckevort, and H.J.F. Knops, Surf. Sci. 424, 109 (1999).

${ }^{14}$ J.A. Jaszczak, W.F. Saam, and B. Yang, Phys. Rev. B 39, 9289 (1989).

${ }^{15}$ H. van Beijeren and I. Nolden, Structure and Dynamics of Surfaces II (Springer, Heidelberg, 1987).

${ }^{16}$ R.H. Swendsen, Phys. Rev. Lett. 37, 1478 (1976).

${ }^{17}$ R.H. Swendsen, Phys. Rev. B 15, 5421 (1977).

${ }^{18}$ S. Wansleben and D.P. Landau, Phys. Rev. B 43, 6006 (1991).

${ }^{19}$ P.J.M. Bastiaansen and H.J.F. Knops, Phys. Rev. E 57, 3784 (1998).

${ }^{20}$ A. Prasad and P.B. Weichman, Phys. Rev. B 57, 4900 (1998).

${ }^{21}$ S. Prestipino and E. Tosatti, Surf. Sci. 377-379, 509 (1997).

${ }^{22}$ J.A. Jaszczak, W.F. Saam, and B. Yang, Phys. Rev. B 41, 6864 (1990).

${ }^{23}$ K. Binder and D. W. Heermann, Monte Carlo Simulation in Statistical Physics (Springer-Verlag, Heidelberg, 1988), pp. 57-59.

${ }^{24}$ G. Mazzeo, E. Carlon, and H. van Beijeren, Surf. Sci. 352-354, 960 (1996)

${ }^{25}$ J.W. Cahn, Acta Metall. 8, 554 (1960). 